\title{
Mobile devices and services: bridging the digital divide in rural areas
}

\author{
Elias Pimenidis* \\ School of Computing, IT and Engineering, \\ University of East London, University Way, \\ London, E16 2RD, UK \\ E-mail: e.pimenidis@uel.ac.uk \\ ${ }^{*}$ Corresponding author
}

\section{Alexander B. Sideridis and Eleni Antonopoulou}

Informatics Laboratory, Agricultural University of Athens, Athens 118 55, Greece

E-mail: as@aua.gr

E-mail: eleni@aua.gr

\begin{abstract}
The digital divide has been mostly affecting the world's poorest primarily due to lack of internet connectivity. A number of e-government services especially built to serve low income citizens do not reach those in need. The rapid expansion of the use of the mobile phone raises hopes that the digital divide can be bridged faster by providing services to people living in rural and remote areas. The authors review successful implementations of secure and trusted e-services available on mobile networks. These could be utilised in addressing the needs of those living in rural areas and are currently digitally marginalised.
\end{abstract}

Keywords: trust; secure mobile services; rural inhabitants; digital divide; mobile payment systems.

Reference to this paper should be made as follows: Pimenidis, E., Sideridis, A.B. and Antonopoulou, E. (2009) 'Mobile devices and services: bridging the digital divide in rural areas', Int. J. Electronic Security and Digital Forensics, Vol. 2, No. 4, pp.424-434.

Biographical notes: Elias Pimenidis is a Senior Lecturer at the University of East London in the UK. The core of his research work focuses on e-business and e-government development projects. His interest in security issues is on online transactions and e-services and in particular, on the effect, these could have on e-government implementations. Other research interests include the evaluation of web services, knowledge management systems and the use of computer games for educational purposes. He is also a Visiting Lecturer at the Informatics laboratory of the Agricultural University of Athens, Greece. 


\begin{abstract}
A.B. Sideridis is Professor in Computer Science and Head of the Informatics Laboratory of the Agricultural University of Athens. He earned his first Degree at the University of Athens and his MSc and $\mathrm{PhD}$ from Brunel University. He has been the project leader of more than 30 successful national and international projects and has published more than 180 scientific papers in management and decision support systems, computer networking related to local and central administration activities, advanced computational numerical modelling, informatics and impact of computers in society and agricultural informatics.

E. Antonopoulou holds a diploma in Plant Science and currently is a $\mathrm{PhD}$ candidate in the Informatics Laboratory of the Agricultural University of Athens. Her doctorate research is focusing to Decision Support Systems design for major field crops.
\end{abstract}

\title{
1 Introduction
}

The digital divide has been a problem that has been identified since the early 1990s when the world started taking considerable steps to improve internet connectivity, and despite the evolution of the World Wide Web -shaping the digital future- the digital divide continues to be an acute problem for most of the Governments. Particularly in Europe, large amounts of money have been spent in an effort to eliminate the effect of the problem.

Literature is full of definitions of the digital divide. It is primarily defined as the degree by which poorer countries lag behind richer ones in terms of accessibility to information technologies and especially the internet. A number of factors, such as: availability of technology, connectivity, literacy and ability to use information systems, determine and characterise its nature. The divide can now be encountered not only among countries but also across different sections of society in the same country and across rural and urban areas (Sukkar, 2002; Bolissian et al., 2006; Sideridis et al., 2006; Karetsos et al., 2007, 2008; Athif and Pimenidis, 2009).

Since the turn of the 21 st century the digital divide has been the subject of many debates, large and high profile initiatives by the United Nations, the World Bank and the IMF, to mention a few. Yet, progress in bridging the gap, to date, has been very slow. Rural areas, in particular, have been more heavily exposed to the divide. Despite considerable investment, land line connectivity in many parts of the world is a privilege that is not enjoyed widely and, in any case, nowhere near the connectivity opportunities available in large urban areas even in the poorest countries in the world. As a result, the digital gap between urban and rural areas remains, if not widens, across many parts of the world and any efforts to bridge the gap between the developed and the developing world are hindered by this disparity (Buys et al., 2008; Athif and Pimenidis, 2009).

In areas where broadband through conventional land line access to the internet has reached users, the gap is slowly but steadily narrowing. However, this assumes that there is considerable land line density available, and in most cases, fibre optic cables, to support high speeds of connection (Economist Intelligence Unit, 2008; Buys et al., 2008). This is achievable and possible in countries in Europe, North America and in other parts of the developed world. A large proportion of the world's population, though, does not 
have access to landlines. Very large percentages of people in Africa and Asia have very limited access to land lines and, often, such lines hardly support any internet connection. Considering the land morphology, cost of investment, financial condition of the citizens and political situation/frequent unrest in these countries, one can acknowledge that developing and maintaining a sound land line infrastructure is very uneconomical, difficult to implement and sustain, and may take many years to complete. Research shows that in a lot of countries affected by the digital divide there are considerably more mobile telephony connections, compared to land lines (Economist Intelligence Unit, 2008).

The authors argue that mobile devices and networks can offer accessibility to e-services to large masses of people who are currently deprived of these due to lack of infrastructure, and are exposed to the ill effects of the digital divide. The rest of the paper is structured as follows. Section 2 explores the requirements of e-readiness as a key factor in the battle to bridge the digital divide and discusses the key contributors where the divide still persists. Section 3 provides an overview of cases where mobile devices and wireless networks have been successfully utilised to overcome the effects of the digital divide. Section 4 discusses the potential of the expanded use of mobile networks as a fix against the divide and addresses the issue of security and trust as the two determinants that might prove to be either drivers or hindrances to such solutions.

\section{E-readiness and factors affecting the spread of the digital divide}

In the current era of unpredictable economic crisis, the digital divide problem is going to further grow and be devastating for quite a few countries for a very simple reason. Governments of the developed countries have already taken economic measures to fight the oncoming unemployment. These measures resulted in heavy investments in industry and particularly the ones that were flourishing till now. On the other hand, poor countries or countries with unstable economies cannot borrow money to invest in infrastructures anymore. On the opposite, their efforts focus on providing certain social benefits guarantying and safeguarding socio-political stability. With no investment, infrastructures remain inefficient and everyone is considering Information and Communications Technology (ICT) investment as a non needed luxury. Without further communication infrastructure investment, internet and the web are offering demanding but not usable eservices.

Over the past few years one of the most reputable publications in the business press, the Economist, has been publishing (in collaboration with IBM) its annual e-readiness rankings and reports. According to Economist Intelligence Unit

\footnotetext{
"E-readiness is a measure of the quality of a country's ICT infrastructure and the ability of its consumers, businesses and governments to use ICT to their benefit. When a country uses ICT to conduct more of their activities, its economy can become more transparent and efficient. The e-readiness rankings also allow governments to gauge the success of their ICT strategies against those of other countries, and provide companies wishing to invest overseas with an overview of the world's most promising investment locations from the perspective of e-readiness." (Economist Intelligence Unit, 2008)
} 
The authors focus not so much on the e-readiness rankings as they do on the data that are linked to the rankings, and this forms part of the study that produces these rankings.

A country's physical communications infrastructure - and the extent to which access infrastructure reaches a majority of people is the fundamental property that determines a country's e-readiness. From the technical point of view this is correct and one could argue that the world is making considerable strides towards improving in this dimension as the number of broadband connections world wide is predicted to reach 1billion by the middle of 2009, from 350 million in 2007 (Economist Intelligence Unit, 2008; Buys et al., 2008). Despite being impressive, these figures show that the majority of the world's population is still without a broadband connection and as the penetration reaches capacity levels in developed countries the progress on that front is going to continue at a much slower rate. Large proportions of the population in extensive areas in Africa and Asia - over $80 \%$ in some cases - do not have access to a land line (Buys et al., 2008). By comparison, the mobile phone networks boasted 1.5 bn mobile subscribers across the world's networks in 2007 and the expectation is that by 2012 the penetration would reach $75 \%$. This is a very ambitious target that might be affected by the current economic climate but, nevertheless, demonstrates the potential of the technology and its ability to extend and expand accessibility to telecommunications, even in the remotest areas.

The potential of the mobile devices as a penetrating factor in remote areas having been established, caution should be taken regarding the cost factor. In developed and developing economies alike, communications services are a basic need, but they must be affordable for the majority of a country's residents in order to achieve a high level of user penetration. Expansion of the user base, in turn, affords individuals and businesses more opportunities to do all the useful things that connectivity offers: to trade, to learn, or even simply to socialise. A supporting super-structure of laws, policies and practices that allows these things to happen in the digital world in accordance with free-market principles is the key requirement for this process to be a success.

In sub-Saharan Africa, in 1993, three-quarters of the countries had no mobile phone network and all existing networks were monopolies. By 2000, all but one had at least one mobile phone network, while 14 of them allowed competition in the markets. By 2006, all had cell networks and only three did not have competitive markets; while 12 of the countries had two or more operators. During the period 2000-2006, the total number of mobile phone subscribers in these countries increased from 10 million to 110 million. The trend in regional subscription shares reflects the proliferation of cell networks. By 2003, South Africa, Mozambique, Zimbabwe, Tanzania, Zambia and Uganda had more cell subscribers than subscribers to fixed lines. While South Africa accounted for $81 \%$ of sub-Saharan subscribers in 2000, its share dropped to 34\% in 2006 (Buys et al., 2008).

Despite the above encouraging figures, researchers are cautious in making the distinction between availability of technology, availability of infrastructure and cost of connectivity. There are still a lot of countries where affordable connectivity is out of reach, even when it comes to mobile telephony. Research ICT Africa (RIA), is a regional academic network that evaluates telecommunications policy. It criticises many African governments for not deregulating markets enough so that competition can bring mobile service pricing down to affordable levels. RIA specifically targets the fixed-to-mobile interconnection rates, which it says are $80-100 \%$ higher than global norms in some of the sub-Saharan countries (Economist Intelligence Unit, 2008). This drives up service costs substantially for mobile users in Africa, who are predominantly poor and promotes a 
considerable market disparity across the area, making it impossible for mobile networks to bridge the connectivity gaps and contribute to the narrowing of the digital divide (Buys et al., 2008).

However, there are others in the ensuing debate in Africa, who argue that market deregulation is not the key issue as, if there is the need and the relevant benefit, poorer people will find ways of securing the best possible connection deal, minimising the cost and, hence, maximising the benefit (RIA, 2009). Although the above view does not completely overcome the issue of cost, it does raise the rather more important question of usability or, even better, the issue of availability of suitable services that would make it worthwhile for even poor people with low levels of education, to be attracted to the use of the mobile phones and communication networks to benefit from internet connectivity.

\section{Solutions based on mobile and wireless networks}

In this section the authors briefly review a few distinctive cases of using innovative technologies to overcome accessibility problems and to an extend attempt to bridge the digital divide. Most of these prove the validity of the argument that mobile devices and wireless networks can provide the basis for viable solutions to addressing the digital divide, but also give rise to a number of issues - security and trust amongst them, which are discussed in Section 4 of the paper.

Ishmael et al. (2008) present a solution based on wireless mesh networks to create a resilient infrastructure. Thus, a local community in the northwest of England had been provided with sufficient broadband access without the need for ground infrastructure. The development has been exciting as it produced very satisfactory results, overcoming the digital gap that was developing in a small community in the heart of a highly developed country. The solution, though, had its limitations as it could only be applied over a small geographical area and it assumed a single point of broadband access through a land line network at a short distance from the initial distribution point. Thus, the potential for expanding and, hence replacing, mobile devices and wide area wireless telephony networks is limited.

Another, decision support service providing application to farmers of central-south India, taking into account existing inadequacies of communication infrastructures, simple in use, is the eSagu system (Reddy and Ankaiah, 2005). It attracts the attention of researchers due to continuous upgrade and extensive use. More specifically, farmers of India are advised by eSagu how to improve their productivity and reduce costs of cultivation. The system provides advices on a regular basis (typically once a week) from sowing to harvesting by storing and processing data, about farmers and their cultivations. eSagu is fully adapted to available network infrastructure supporting farmers familiar or not with ICT.

Ramamritham et al. (2008) discuss the case of 'The aAQUA Approach', a discussion forum that provides answers to questions related to agriculture and animal husbandry, in rural India. The system can receive and respond to queries via internet access or text messaging via mobile phones. It targets users that are predominately semi-literate, non-English speakers that are, at best, novice users of the internet. Although the content appears to be rich and useful, access is limited to those who can access the full internet service and can also afford to pay for it. Experts provide answers within $36 \mathrm{~h}$ and archives are continuously updated. Consequently, this can be beneficial for the user in a 
remote rural area with limited access to expert resources and a mobile phone connection. This example demonstrates the potential of the mobile phone to be combined with web technologies to allow for limited interaction and, potentially, a rather slow response. It does partly overcome the lack of connectivity and possibly the limited ability to use the internet effectively by people who have a low level of education.

Fink et al. (2008) present the case of using Power Line Carrier technology to transmit data signals through an existing power line infrastructure where a proper landline based telecommunications infrastructure is not available to support broadband access. This method appears to be very efficient in tackling the long distance problem of connection, but does not address the access points for individuals. There are Wi-Fi systems that have to be employed to allow individuals to connect to the internet and access online services, but these have limited range of access and need to be quite densely set up and closely located to the long distance transmission medium. Longer distances can be achieved by WiMax which offers connection to distances of up to $10 \mathrm{~km}$. The limitation here is the need to develop a fibre-optic based backbone for WiMax radio. On the other hand, $3 \mathrm{G}$ and mobile networks are available virtually in every populated area due to the cellular nature of their networks and the very high popularity of the mobile phone in the regions of Brazil where this particular case has been applied.

This case has, potentially, great applicability in countries with a good electrical power distribution infrastructure when they are seeking to provide internet accessibility at a reduced cost. However, when the poorer countries of this world are considered, this solution might not be practical enough as there are vast areas where electricity is not broadly available. This might cause an oxymoron as to the use of mobile phones, but it is quite common in poor areas in Africa and Asia to have better mobile phone networks than electrical power distribution grids.

Parikh (2005) raised a different question and in 2005 discusses a system whereby the use of a smartphone, and people with minimal digital information skills, manipulate traditional documents in rural areas in India. The basic problem with any system offering a connectivity solution to rural areas is to introduce computing seamlessly into a physical, natural world populated by non-expert users who 'cannot', 'do not', or would 'rather not' spend their time in front of computers. These users are not knowledge workers, do not use modern information technology as part of their daily lives, and would like maximal utility from computing devices with minimal engagement. Paper has several advantages. Since their daily lives are packed with physical tasks, rural users are much more comfortable with artefacts they can handle and touch. Paper affords a physical representation of information that can be communally viewed, edited, and stored. In some cases, illiterate users have even learned to interpret data from paper forms. Traditional paper based documents are an information medium that the local people own and trust, and they provide a greater sense of security than rented or borrowed appliances such as PCs or even mobile devices to access digital devices. Companies have developed commercial document-processing systems that recognise structure and text from scanned paper forms and integrate them with back-end data sources, while other researchers have developed visual codes to embed camera-scannable digital data in physical documents. All these efforts attempt to integrate it with a digital world that can index, search, and retrieve information with great flexibility. 
Parikh and colleagues developed an information services architecture that uses a smartphone equipped with a built-in digital camera to process augmented paper documents. CAM, so called because the phone's camera plays a key role in the user interface, is a three-tiered, document-based architecture for providing remote rural information services. The mobile phone acts as a primary data-capture and - entry device in a document-processing system. It also utilises the CAM framework, including CamShell - a phone-scannable scripting language that can encode a user interface along with processing and routing instructions within paper documents.

Through this system, rural users transfer paper-based information to roving smartphone middleware, which uploads the data to remote storage for distribution in various ways. Familiarity with the technology involved (mobile phone) builds trust into the system, which simply handles the traditional medium of communication (paper based documents) in a more modern way.

The CamBrowser application executes user instructions until it encounters a network or application request. The request can be one of three types: a URL, a phone call, or an MMS message. The interpreter executes the request using the appropriate application, with the local variables as the request content.

In the case of a URL, the phone can operate in one of three network modes:

- Online, in which the phone executes HTTP requests over an internet connection.

- Offline, in which the phone caches requests locally and executes them later when it has connectivity. This is facilitated either via a short-range (Bluetooth) or long-range (internet) connection.

- Proxy, in which the phone acts as a proxy interface for a nearby PC, forwarding requests over a Bluetooth connection. The PC's web browser processes and displays these requests.

These network modes let users choose where and how to incur communications costs. If they live in an area where the mobile phone network is not good, or if service costs are disproportionately high, they can physically carry the phone to a better connected location.

Users can view the response document on the phone's screen, on a nearby PC, or printed from a connected printer. Response screens can include further CamShell scripts, which users can scan from the screen or from a printed copy.

The scripts can include scannable links to further content or can act like wizards that construct new CamForms based on prior input.

The CAM architecture's distributed nature makes security an important concern. CamShell provides instructions for performing encryption, digital signing, or both (signcryption). These instructions include digital keys and can be embedded either directly in CamForms or within special security tokens, called CamTokens. When the CamBrowser processes these instructions, the software automatically signs and/or encrypts the collected data before transmission.

Remembered tokens such as passwords and PINs have are considered as being inflexible and insecure, especially when used by casual users like the ones on rural areas. Conversely, humans have a long history of trust practices built around exchanging physical tokens (for example, money). As a result, the physical keys as implemented in CamShell, might be more usable in rural areas because they carry more information 
and most rural users are unlikely to remember long or unfamiliar combinations of letters, and their remembered passwords would be vulnerable to cracks. Physical tokens can carry much more data and would be easier to replace if compromised.

Users can enter them securely. Rural users are unlikely to have the capacity of unfettered access to enter a conventional password or PIN without others observing it. Users can explicitly share and distribute them. Physical tokens can have an arbitrary non-zero duplication cost; so users can distribute, lend, or share them with fixed risk.

Trust is another important consideration in the CAM user interface's design. In most cases, CamForm users will not have direct access to a mobile phone and will, therefore, have to rely on local phone-equipped agents to capture and transmit their data.

Pentland et al. (2004) discuss DakNet, an ad-hoc network that uses wireless technology, to provide asynchronous digital connectivity. This has been developed by MIT Media Lab researchers and has been successfully deployed in remote parts of both India and Cambodia at a cost two orders of magnitude less than that of traditional landline solutions. Villagers now get affordable internet services - and they are using them. The DakNet wireless network takes advantage of the existing communications and transportation infrastructure to distribute digital connectivity to outlying villages lacking a digital communications infrastructure. It combines a physical means of transportation with wireless data transfer to extend the internet connectivity that a central uplink or hub, such as a cybercafe, VSAT system, or post office provides. Instead of trying to relay data over a long distance, which can be both expensive and power hungry, DakNet transmits data over short point-to-point links between kiosks and portable storage devices, called Mobile Access Points (MAPs). Mounted on, and powered by, a bus, a motorcycle, or even a bicycle with a small generator, a MAP physically transports data among public kiosks and private communications devices (as an intranet) and between kiosks and a hub (for non-real-time internet access). Low-cost $\mathrm{WiFi}$ radio transceivers automatically transfer the data stored in the MAP at high bandwidth for each point-to-point connection.

It's usefulness is limited to small geographical locations, but the conventional ways of 'moving' data makes it more 'familiar-like' to the users and hence the high levels of trust and popularity it has attained. Finally, the fact that data are transported, and not transmitted, over long distances makes the case for security more simple and the acceptance on behalf of the user easier, leading to users engaging in a number of e-services available.

Finally, one of the most advanced and innovative applications involving the use of mobile devices in advancing the quality of life of users by offering e-services is a novel payment method applied in Africa. This involves the transfer of air-time that the user has paid for with real money at a physical location. The environment is secure and there is a transaction history maintained in the mobile phone provider's archives. Apart from the obvious use of electronic transactions that the user can complete, the transaction history can be used as proof of credit worthiness when the user deals with banks and other financial institutions. This is particularly useful as a large number of Africans do not have a bank account nor do they own a property to use as collateral when dealing with a bank for the purposes of a loan (Donner et al., 2008). 


\section{Trust and security in mobile services}

Most of the cases reviewed discuss the issue of access to the internet and services without any major concerns to security issues. The reason is that in most examples considered in the previous section access has been only simple information retrieval without the involvement of any exchange of critical or sensitive data. In most cases the concern has been that of earning user's trust in the systems and services so that the public is comfortable with the idea of using them.

Experience so far has shown that the users, even the poorer amongst them, are willing to pay for services if these will mean less travel, less waiting time and less corruption in completing a transaction or service (Pentland et al., 2004; Sohoo, 2008). Therefore, the first issue is that the availability of service in a form that can be completed with the technology available and the levels of literacy the users possess. Thus, technology needs to accommodate the users' literacy levels in order to get them online, if any e-inclusion is to be achieved. This is rather in contrast to the current belief that countries have to embark on massive educational programmes to make their populations computer-literate. In most cases of the countries that have been digitally marginalised this is almost impossible to achieve, as these populations will be drawn even deeper into digital exclusion (Economist Intelligence Unit, 2008). Providing simple solutions based on familiar technological tools will earn the users' trust and will make it simpler for the providers to explain any risks and implement any security measures that are simple to follow. The example of transactions based on transfer of air-time discussed in the previous section is one that epitomises this approach. The concepts dealt with are very simple, easily comprehensible by the user and provide adequate security to facilitate financial transactions in areas where banking practices are not as secure as in the developed world and people do not trust banks.

Financial services provision is particularly ripe for collaboration initiatives but the big financial institutions have not invested heavily on mobile banking in the developed world as this is not a priority market for them. In poor countries though, mobile banking is increasingly becoming the primary channel for retail banks to reach their customers. This, coupled with the use of biometrics, has been making inroads as a tool for private organisations and governments to increase access to online services. India is ahead in using biometric technology to target underserved communities - and using readily available, practical technology to do so. In this way the mobile phone provides a secure and easy to use system for legal transactions encompassing the trust of people. The initiative for the provision of biometric security features has to be government led to allow for the sensitive issue of privacy of personal data. The right legal framework is essential in this case to establish the environment and policies according to which such data are to be captured, stored and used to instil confidence in the public that participates in such schemes (Mousavi et al., 2008; Sohoo, 2008). The Department of Rural Development in India's state of Andhra Pradesh is implementing a programme where private customer-service providers issue social security payments to citizens using mobile phones loaded with banking applications which, in turn, communicate with biometric smartcards via Radio-Frequency Identification (RFID) to validate the applicants' details. The cards will soon be extended to allow holders to make other transactions such as housing payments or small farm loans (Economist Intelligence Unit, 2008). 


\section{Conclusions}

Mobile devices are a familiar tool for a large proportion of the world's population. The use of the mobile phone has surpassed the availability and use of landlines and is continuing to expand at a much more rapid pace. This makes it the obvious choice as point of access to the internet and e-services for areas that have so far been digitally marginalised due to the effects of the digital divide.

Appropriate applications and services have been implemented and have been proved to provide secure and trusted environments for large masses of people to utilise them irrespective of literacy levels and internet usage expertise. It is up to the individual governments and international organisations to promote such telecommunications networks and electronic services to help these marginalised populations to reap some of the benefits of the digital world as it is shaping in the 21 st century.

\section{References}

Athif, A. and Pimenidis, E. (2009) 'E-crime threats on e-government - the case of Maldives', Proceedings of the 4th Advances in Computing and Technology Conference (AC \& T'09), 27 January, School of Computing, Information Technology and Engineering, University of East London, UK, pp.16-25.

Bolissian, J., Pimendis, E., Liadis, L. and Andreopoulou, Z. (2006) 'E-readiness or digital exclusion - evaluating a country's status', Proceedings of the 2nd E-Democracy National Conference with International Participation, 16-17 March, Athens Bar Association and the Scientific Council for the Information Society, Athens, Greece, pp.87-96.

Buys, P., Dasgupta, S., Thomas, T. and Wheeler, D. (2008) Determinants of a Digital Divide in Sub-Saharan Africa: A Spatial Econometric Analysis of Cell Phone Coverage, The World Bank Development Research Group, Sustainable Rural and Urban Development Team, Washington DC, USA.

Donner, J., Omwansa, T., Moore, J., Richardson, B. and Comninos, A. (2008) M-Banking and M-Payments for Social Impact, Mobile Active 08, www.MobileActive.org (accessed 25/04/2009).

Economist Intelligence Unit (2008) The Economist, The IBM Institute for Business Value (2009), E-readiness rankings 2008.

Fink, D., Zo, H. and Rho, J.J. (2008) 'The long tail of loop distance for broadband over power lines: finding a new niche for rural telecommunications in Brazil', Proceedings of the 2008 Third International Conference on Convergence and Hybrid Information Technology, IEEE Computer Society, Busan, Korea, pp.980-984.

Ishmael, J., Bury, S., Pezaros, D. and Race, N. (2008) 'Deploying rural community wireless mesh networks', IEEE Internet Computing, Vol. 12, No. 4, pp.22-29.

Karetsos, S., Costopoulou, C., Sideridis, A.B., Patrikakis, C. and Koukouli, M. (2007) 'Bio@gro - an online multilingual organic agriculture e-services platform', Information Services and Use, IOS Press, Vol. 27, pp.123-132.

Karetsos, S., Costopoulou, C. and Ntaliani, M. (2008) 'Building a virtual community for organic agriculture', International Journal of Web-Based Communities, Interscience Publishing, Vol. 4, No. 3, pp.366-383.

Mousavi, S.A.A., Pimenidis, E. and Jahankhani, H. (2008) 'Cultivating trust - an e-government development model for addressing the needs of developing countries', International Journal of Electronic Security and Digital Forensics (IJESDF), Vol. 1, No. 3, pp.233-248.

Parikh, T.S. (2005) 'Using mobile phones for secure, distributed document processing in the developing world', IEEE Pervasive Computing, Vol. 4, No. 2, pp.74-81. 
Pentland, A., Fletcher, R. and Hasson, A. (2004) 'DakNet: rethinking connectivity in developing nations', IEEE Computer, Vol. 37, No. 1, pp.78-83.

Ramamritham, K., Bahuman, A., Sahni, S., Baru, M., Bahuman, C., Chandran, A. and Joshi, M. (2008) 'The aAQUA approach: innovative web 2.0 tools for developing countries', IEEE Internet Computing, Vol. 12, No. 2, pp.62-70.

Research ICT Africa (RIA) (2009) Government Regulation Irrelevant in Telecommunications, www.ResearchICTAfrica.net (accessed 25/04/2009).

Sohoo, S. (2008) 'ICT initiative of SAARC agriculture centre in the SAARC region', Proceedings of the 2008 International Conference on Computer Science and Information Technology, IEEE Computer Society, Beijing, China, pp.923-929.

Sukkar, N. (2002) 'The digital divide and development', Proceedings of the Mediterranean Development Forum, 6-9 October, Amman, Syria, pp.1-10. 